\title{
SEIBERG-WITTEN INVARIANTS OF GENERALISED RATIONAL BLOW-DOWNS
}

\author{
JONGIL PARK
}

\begin{abstract}
One of the main problems in Seiberg-Witten theory is to find (SW)-basic classes and their invariants for a given smooth 4 -manifold. The rational blow-down procedure introduced by Fintushel and Stern is one way to compute these invariants for some smooth 4-manifolds. In this paper, we extend their results to the general case. That is, we find (SW)-basic classes and Seiberg-Witten invariants for generalised rational blow-down 4-manifolds by using index computations.
\end{abstract}

\section{INTRODUCTION}

As gauge theory (Donaldson theory and Seiberg-Witten theory) is developed, the fundamental problem in this area is to find its invariants for a given smooth 4-manifold.

In 1993, Fintushel and Stern introduced a surgical procedure, called rational blowdown, to compute the Donaldson series for simply connected regular elliptic surfaces with multiple fibres of relatively prime orders. 'Rational blow-down' means that if a smooth 4-manifold $X$ contains a certain configuration $C_{p}$ of transversally intersecting 2-spheres whose boundary is $L\left(p^{2}, 1-p\right)$, then one can construct a new smooth 4manifold $X_{p}$ from $X$ by replacing $C_{p}$ with a rational ball $B_{p}$.

In fact, Casson and Harer [2] showed that for any pair of relatively prime integers $p$ and $q, L\left(p^{2}, 1-p q\right)$ bounds a rational ball $B_{p, q}$. Hence one can extend this rational blow-down procedure to the general case, that is, whenever a smooth 4-manifold $X$ contains a certain configuration $C_{p, q}$ of transversally intersecting 2 -spheres whose boundary is $L\left(p^{2}, 1-p q\right)$, one can always construct a new smooth 4-manifold $X_{p, q}$ by replacing $C_{p, q}$ with a rational ball $B_{p, q}$.

For the $q=1$ case, Fintushel and Stern initially computed the Donaldson series of $X_{p}=X_{p, 1}$ from the Donaldson series of $X$, and later they computed the SeibergWitten invariants of $X_{p}[5]$. In Section 3 of this paper we extend these results to the general case. Explicitly, we prove the following theorem by using index computations:

Received 4th December, 1996.

This paper originally appeared in the author's Ph.D. thesis at Michigan State University. The author would like to thank Professor Ronald Fintushel for suggesting this problem, and for his guidance and help while working on this problem.

Copyright Clearance Centre, Inc. Serial-fee code: 0004-9729/97 $\$ A 2.00+0.00$. 
Theorem 1.1. Suppose $X$ is a smooth 4-manifold which contains a configuration $C_{p, q}$. If $L$ is a characteristic line bundle on $X$ such that $S W_{X}(L) \neq 0$, $\left(\left.L\right|_{C_{p, q}}\right)^{2}=-b_{2}\left(C_{p, q}\right)$ and $c_{1}\left(\left.L\right|_{L\left(p^{2}, 1-p q\right)}\right)=m p \in \mathbf{Z}_{p^{2}} \cong H^{2}\left(L\left(p^{2}, 1-p q\right) ; \mathbf{Z}\right)$ with $m \equiv(p-1)(\bmod 2)$, then $L$ induces a characteristic line bundle $\bar{L}$ on $X_{p, q}$ such that $S W_{X_{p, q}}(\bar{L})=S W_{X}(L)$.

Furthermore, we prove the following theorem:

THEOREM 1.2. If a simply connected smooth 4-manifold $X$ contains a configuration $C_{p, q}$ satisfying condition (*) below, then the $S W$-invariants of $X_{p, q}$ are completely determined by those of $X$. That is, for any characteristic line bundle $\bar{L}$ on $X_{p, q}$ with $S W_{X_{p, q}}(\bar{L}) \neq 0$, there exists a characteristic line bundle $L$ on $X$ such that $S W_{X}(L)=S W_{X_{p, q}}(\bar{L})$.

The condition $(*)$ in the theorem above is the following:

$$
\text { (*) } \begin{aligned}
\left\{\partial\left(\left.\sum_{i=1}^{k} \varepsilon_{i} e_{i}\right|_{B_{p, q}}\right)\right. & \left.: \varepsilon_{i}= \pm 1, \forall i\right\} \\
& =\{m p:-(p-1) \leqslant m \leqslant(p-1) \text { and } m \equiv(p-1)(\bmod 2)\}
\end{aligned}
$$

All known configurations $C_{p, q}$ satisfy this condition.

\section{The Topology of Rational Blow-Downs}

In this section we describe topological aspects and several examples of rational blow-down 4-manifolds. For any relatively prime integers $p$ and $q$ with $1 \leqslant q<p$, we define a configuration $C_{p, q}$ as a smooth 4 -manifold obtained by plumbing disk bundles over the 2-sphere instructed by the following linear diagram

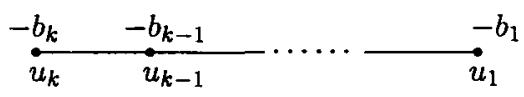

where $p^{2} /(p q-1)=\left[b_{k}, b_{k-1}, \ldots, b_{1}\right]$ is a unique continued linear fraction with all $b_{i} \geqslant 2$, and each vertex $u_{i}$ represents a disk bundle over the 2 -sphere whose Euler number is $-b_{i}$. Then the configuration $C_{p, q}$ has the following properties:

1. It is a simply connected smooth 4-manifold whose boundary is the lens space $L\left(p^{2}, 1-p q\right)$.

2. $H_{2}\left(C_{p, q} ; \mathbf{Z}\right) \cong \bigoplus_{i=1}^{k} \mathbf{Z}$ has generators $\left\{u_{i}: 1 \leqslant i \leqslant k\right\}$ which can be represented by embedded 2 -spheres, that is, each $u_{i}$ is represented by the zero-section $S_{i}^{2}$ of the disk bundle $u_{i}$ over $S^{2}$. (We use $u_{i}$ for both a generator and the corresponding disk bundle.) 
3. The plumbing matrix for $C_{p, q}$ with respect to the basis $\left\{u_{i}: 1 \leqslant i \leqslant k\right\}$ is given by the symmetric $k \times k$ matrix

$$
P=\left(\begin{array}{cccccc}
-b_{1} & 1 & 0 & & & \\
1 & -b_{2} & 1 & & 0 & \\
0 & 1 & -b_{3} & & & \\
& & & \ddots & & \\
& 0 & & & -b_{k-1} & 1 \\
& & & & 1 & -b_{k}
\end{array}\right)
$$

so that $C_{p, q}$ is negative definite.

4. The intersection form on $H^{2}\left(C_{p, q} ; \mathbf{Z}\right)$ with respect to the dual basis $\left\{\gamma_{i}\right.$ : $1 \leqslant i \leqslant k\}$ (that is, $\left\langle\gamma_{i}, u_{j}\right\rangle=\delta_{i j}$ ) is given by

$$
Q:=\left(\gamma_{i} \cdot \gamma_{j}\right)=P^{-1}
$$

Proof: Note that the intersection form $Q$ on $H^{2}\left(C_{p, q} ; \mathbf{Z}\right)$ is defined by

$$
\gamma_{i} \cdot \gamma_{j}:=\frac{1}{p^{2}}\left\langle\gamma_{i}, P D \gamma_{j}^{\prime}\right\rangle
$$

where $\gamma_{j}^{\prime} \in H^{2}\left(C_{p, q}, \partial C_{p, q} ; \mathbf{Z}\right)$ is determined by $j^{*}\left(\gamma_{j}^{\prime}\right)=p^{2} \cdot \gamma_{j}$ in the sequence

$$
0 \longrightarrow H^{2}\left(C_{p, q}, \partial C_{p, q} ; \mathbf{Z}\right) \stackrel{\jmath^{*}}{\longrightarrow} H^{2}\left(C_{p, q} ; \mathbf{Z}\right) \stackrel{\partial}{\longrightarrow} H^{2}\left(\partial C_{p, q} ; \mathbf{Z}\right) \longrightarrow 0
$$

Since $\jmath^{*}=P$, we have

$$
\begin{aligned}
\gamma_{i} \cdot \gamma_{j} & :=\frac{1}{p^{2}}\left\langle\gamma_{i}, P D \gamma_{j}^{\prime}\right\rangle=\frac{1}{p^{2}}\left\langle\gamma_{i}, P^{-1}\left(p^{2} \cdot P D \gamma_{j}\right)\right\rangle=\left\langle\gamma_{i}, P^{-1}\left(P D \gamma_{j}\right)\right\rangle \\
& =\left(P^{-1}\right)_{i j}
\end{aligned}
$$

LEMMA 2.1. The inclusion induced homomorphism $\partial: H^{2}\left(C_{p, q} ; \mathbf{Z}\right) \longrightarrow$ $H^{2}\left(\partial C_{p, q} ; \mathbf{Z}\right) \cong \mathbf{Z}_{p^{2}}$ is given by $\partial\left(\gamma_{i}\right)=n_{i}$, where $n_{i}$ is a number satisfying

$$
\left(\begin{array}{c}
* \\
n_{i}
\end{array}\right):=\left(\begin{array}{cc}
-1 & 0 \\
b_{1} & 1
\end{array}\right)\left(\begin{array}{ll}
0 & 1 \\
1 & 0
\end{array}\right)\left(\begin{array}{cc}
-1 & 0 \\
b_{2} & 1
\end{array}\right) \cdots\left(\begin{array}{cc}
-1 & 0 \\
b_{i-1} & 1
\end{array}\right)\left(\begin{array}{ll}
0 & 1 \\
1 & 0
\end{array}\right)\left(\begin{array}{l}
0 \\
1
\end{array}\right) \text {. }
$$

Proof: By Poincaré duality, it suffices to show $\partial: H_{2}\left(C_{p, q}, \partial C_{p, q} ; \mathbf{Z}\right) \rightarrow H_{1}\left(\partial C_{p, q} ; \mathbf{Z}\right)$ is given by $\partial\left(P D \gamma_{i}\right)=n_{i}$. For each $i$, choose a fibre $D_{i}^{2}$ of a disk bundle $u_{i}$ over $S^{2}$ 
so that $D_{i}^{2} \cdot S_{j}^{2}=\delta_{i j}$. Then $D_{i}^{2}$ is a representative for $P D\left(\gamma_{i}\right) \in H_{2}\left(C_{p, q}, \partial C_{p, q} ; \mathbf{Z}\right)$. Since

$$
\begin{aligned}
\partial C_{p, q} & =D^{+} \times S_{k}^{1} \cup_{A_{k}} \partial D^{-} \times S_{k}^{1} \cup_{B} \partial D^{+} \times S_{k-1}^{1} \cup_{A_{k-1}} \cdots \cup_{A_{1}} D^{-} \times S_{1}^{1} \\
& =D^{+} \times S_{k}^{1} \cup_{A} D^{-} \times S_{1}^{1}
\end{aligned}
$$

where $S_{i}^{1}:=\partial D_{i}^{2}$ and $A:=A_{k} B A_{k-1} \cdots A_{1}$ with $A_{i}:=\left(\begin{array}{cc}-1 & 0 \\ b_{i} & 1\end{array}\right)$, and $B:=$ $\left(\begin{array}{ll}0 & 1 \\ 1 & 0\end{array}\right)$ we have

$$
\begin{aligned}
\partial\left(P D \gamma_{i}\right) & =\partial\left(D_{i}^{2}\right) \\
& =\left(\begin{array}{ll}
-1 & 0 \\
b_{1} & 1
\end{array}\right)\left(\begin{array}{ll}
0 & 1 \\
1 & 0
\end{array}\right) \cdots\left(\begin{array}{cc}
-1 & 0 \\
b_{i-1} & 1
\end{array}\right)\left(\begin{array}{ll}
0 & 1 \\
1 & 0
\end{array}\right)\left(\begin{array}{l}
0 \\
1
\end{array}\right)=\left(\begin{array}{c}
* \\
n_{i}
\end{array}\right)
\end{aligned}
$$

which is homologous to $\left(\begin{array}{c}0 \\ n_{i}\end{array}\right)$ in $H_{1}\left(\partial C_{p, q} ; \mathbf{Z}\right)$. Hence, by choosing $\left(\begin{array}{l}0 \\ 1\end{array}\right)$ as a generator of $H_{1}\left(\partial C_{p, q} ; \mathbf{Z}\right)$, we have $\partial\left(P D \gamma_{i}\right)=n_{i}$.

LEMma 2.2. The lens space $L\left(p^{2}, 1-p q\right)=\partial C_{p, q}$ bounds a rational ball $B_{p, q}$ with $\pi_{1}\left(B_{p, q}\right)=\mathbf{Z}_{p}$, and the inclusion induced homomorphism

$$
\iota^{*}: H^{2}\left(B_{p, q} ; \mathbf{Z}\right) \cong \mathbf{Z}_{p} \longrightarrow H^{2}\left(L\left(p^{2}, 1-p q\right) ; \mathbf{Z}\right) \cong \mathbf{Z}_{p}
$$

can be given by $n \longmapsto n p$.

Proof: The first part was proved by Casson and Harer [2]. For the second part, since the Mayer-Vietoris sequence for $X \equiv C_{p, q} \cup_{L} \overline{B_{p, q}}$ which is homeomorphic to $\$ k \overline{\mathbf{C P}}^{2}$

$$
0 \longrightarrow H_{2}\left(C_{p, q} ; \mathbf{Z}\right) \oplus H_{2}\left(B_{p, q} ; \mathbf{Z}\right) \longrightarrow H_{2}\left(\sharp k \overline{\mathbf{C P}}^{2} ; \mathbf{Z}\right) \longrightarrow \cdots
$$

implies $H_{2}\left(B_{p, q} ; \mathbf{Z}\right)$ is torsion free, by Poincaré duality, $H^{2}\left(B_{p, q}, \partial B_{p, q} ; \mathbf{Z}\right) \cong H_{2}\left(B_{p, q}\right)=$ 0 . On the other hand, since the exact sequence for $\left(B_{p, q}, \partial B_{p, q}\right)$ also implies that

$$
\iota^{*}: H^{2}\left(B_{p, q} ; \mathbf{Z}\right) \cong \mathbf{Z}_{p} \longrightarrow H^{2}\left(\partial B_{p, q} ; \mathbf{Z}\right) \cong \mathbf{Z}_{p^{2}}
$$

is injective, $\iota^{*}(1)=l p$ for some $l$ with $\operatorname{gcd}(l, p)=1$. Hence, by re-choosing a generator of $H^{2}\left(\partial B_{p, q} ; \mathbf{Z}\right) \cong Z_{p^{2}}$, we may assume that $\iota^{*}(1)=p$, so that $\iota^{*}(n)=n p$.

Lemma 2.3. $B_{p, q}$ is spin if $p$ is odd, and $B_{p, q}$ is not spin if $p$ is even.

Proof: If $p$ is odd, then $H_{1}\left(B_{p, q}\right) \cong \mathbf{Z}_{p}$ implies $H^{2}\left(B_{p, q} ; \mathbf{Z}_{2}\right) \cong \operatorname{Ext}\left(H_{1}\left(B_{p, q}\right) ; \mathbf{Z}_{2}\right)$ $=0$. Assume $p$ is even and $B_{p, q}$ is spin. Then the index of the Dirac operator on $B_{p, q}$ should be an integer. But the index computation on $B_{p, q}$ (Proposition 3.3 and its remark) shows that it is not an integer-a contradiction! 
Now we define the rational blow-down procedure: Suppose $X$ is a smooth 4 manifold which contains a configuration $C_{p, q}$ for some relatively prime integers $p$ and $q$. We construct a new smooth 4-manifold $X_{p, q}$, called the rational blow-down of $X$, by replacing $C_{p, q}$ with the rational ball $B_{p, q}$ (Figure 1). We call this procedure a '(generalised) rational blow-down'. Note that this procedure is well defined, that is, $X_{p, q}$ is uniquely constructed (up to diffeomorphism) from $X$ because each diffeomorphism of $\partial B_{p, q}=L\left(p^{2}, 1-p q\right)$ extends over the rational ball $B_{p, q}$ by the same argument as in [5, Corollary 2.2].

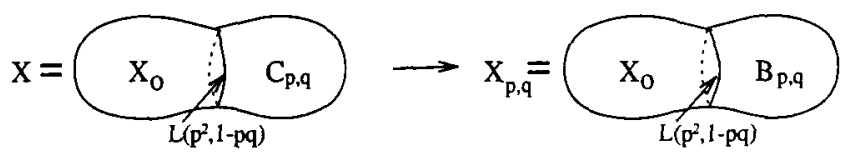

Figure 1

LEMma 2.4. $b^{+}\left(X_{p, q}\right)=b^{+}(X)$ and $c_{1}^{2}\left(X_{p, q}\right)=c_{1}^{2}(X)+k$, where $k=b_{2}\left(C_{p, q}\right)$.

Proof: Since $C_{p, q}$ is negative definite, $b^{+}\left(X_{p, q}\right)=b^{+}(X)$ and

$$
\begin{aligned}
c_{1}^{2}\left(X_{p, q}\right) & =3 \sigma\left(X_{p, q}\right)+2 e\left(X_{p, q}\right) \\
& =3(\sigma(X)+k)+2(e(X)-k) \\
& =c_{1}^{2}(X)+k .
\end{aligned}
$$

where $\sigma(X)$ is the signature of $X$ and $e(X)$ is the Euler characteristic of $X$.

Here are several configurations $C_{p, q}$ that will be used later.

CASE $q=1$. This case is studied in [5], whose configuration $C_{p, 1}$ is

$$
\begin{aligned}
-(p+2) \quad-2 & \\
\stackrel{\bullet}{u_{p-1}} & u_{p-2}
\end{aligned} \cdots \cdots \frac{-2}{a_{1}}
$$

Fintushel and Stern used this configuration to show that the rational blow-down of $E(n) \sharp(p-1) \overline{\mathbf{C P}}^{2}$ is diffeomorphic to $E(n ; p), p$-log transform on $E(n)$, and to compute the Donaldson and Seiberg-Witten invariants of simply connected elliptic surfaces with multiple fibres. Here $E(n)$ is a simply connected elliptic surface with no multiple fibres and holomorphic Euler characteristic $n$, and ' $p$-log transform on $E(n)$ ' is the result of removing a tubular neighbourhood of a torus fibre in $E(n)$, say $T^{2} \times D^{2}$, and regluing it by a diffeomorphism

$$
\varphi: T^{2} \times \partial D^{2} \longrightarrow T^{2} \times \partial D^{2}
$$

such that the absolute value of the degree of the map

$$
\operatorname{proj}_{\partial D^{2}} \circ \varphi: p t \times \partial D^{2} \longrightarrow \partial D^{2}
$$


is $p$. Note that ' $p$ - $\log$ transform on $E(n)$ ' is well defined, that is, $E(n ; p)$ is uniquely determined up to diffeomorphism by the fact that if $\operatorname{proj}_{\partial D^{2}} \circ \varphi$ and $\operatorname{proj}_{\partial D^{2}} \circ \varphi^{\prime}$ have the same degree up to sign, then the resulting two manifolds are diffeomorphic [6, Proposition 2.1].

CASE $p=k q-1(k, q \geqslant 2)$. We assume $q \geqslant 3$ (the $q=2$ case is also obtained in a similar way). The configuration $C_{p, q}$ is given by

which can be embedded in $\sharp(k+q-2) \overline{\mathbf{C P}}^{2}$ by choosing

$$
u_{i}:= \begin{cases}e_{k+q-2-i}-e_{k+q-1-i} & i=1, \ldots, k-2 \\ e_{q-2}-e_{q-1}-e_{q} & i=k-1 \\ e_{k+q-3-i}-e_{k+q-2-i} & i=k, \ldots, k+q-4 \\ -2 e_{1}-e_{2}-\cdots-e_{q-1} & i=k+q-3 \\ e_{q-1}-e_{q}-\cdots-e_{k+q-2} & i=k+q-2\end{cases}
$$

where each $e_{i}(1 \leqslant i \leqslant k+q-2)$ is the exceptional divisor in $\sharp(k+q-2) \overline{\mathbf{C P}}^{2}$. Furthermore, by using Lemma 2.1 , we get its boundary values

$$
\partial \gamma_{i}= \begin{cases}i & i=1, \ldots, k-1 \\ (i+2-k) k-i & i=k, \ldots, k+q-3 \\ p q-1 & i=k+q-2\end{cases}
$$

which imply that $C_{k q-1, q}$ satisfies the condition (*) mentioned in the introduction.

THEOREM 2.1. For any integers $k$ and $q(k, q \geqslant 2)$, there is an embedding $C_{k q-1, q} \subset E(n) \sharp(k+q-2) \overline{\mathbf{C P}}^{2}$ such that the rational blow-down is diffeomorphic to $E(n ; k q-1)$.

Proof: Consider the homology class $f$ of the fibre in $E(n)$ which can be represented by an immersed 2 -sphere with one positive double point and self-intersection 0 (a nodal fibre). Blow up this double point so that $f-2 e_{1}\left(e_{1}\right.$ is the exceptional divisor) is represented by an embedded sphere. Since $e_{1}$ intersects $f-2 e_{1}$ at two positive points, blow up one of these points again. By continuing in this way, we get a configuration $C_{k q-1, q}$ in $E(n) \sharp(k+q-2) \overline{\mathbf{C P}}^{2}$. We draw the case $q \geqslant 3$ (Figure 2) (the $q=2$ case is similar). The claim that the rational blow-down of $E(n) \sharp(k+q-2) \overline{\mathbf{C P}}^{2}$ is diffeomorphic to $E(n ; k q-1)$ can be proved by Kirby calculus on the neighbourhood of a cusp fibre as in [5, Theorem 3.1]. 

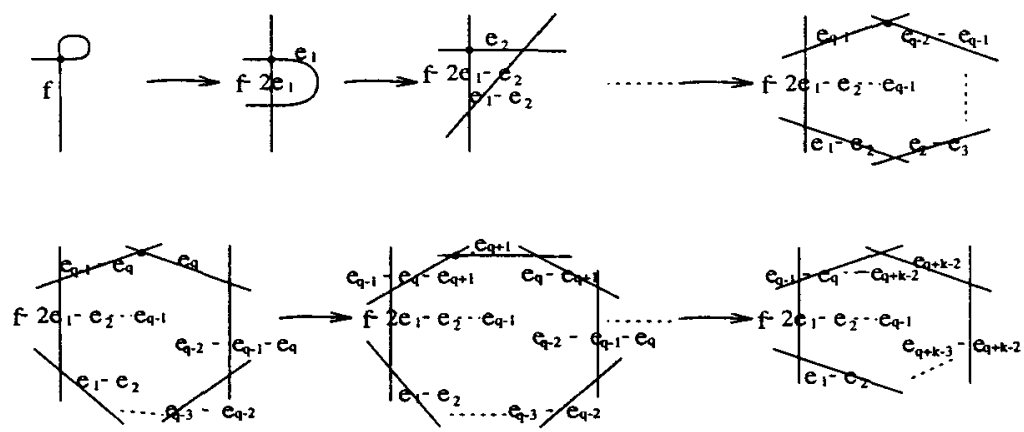

Figure 2

Here are a few remarks on this theorem:

1. The theorem implies that there are many ways to obtain $E(n ; p), p$-log transform on $E(n)$, from $E(n)$ via a rational blow-down procedure; so one can choose an 'economical' way to get $E(n ; p)$. For example, $E(n, 11)$ is diffeomorphic to the rational blowdown of $C_{11,1} \subset E(n) \sharp 10 \overline{\mathbf{C P}}^{2}$, of $C_{11,2} \subset E(n) \sharp 6 \overline{\mathbf{C P}}^{2}$, and of $C_{11,3} \subset E(n) \sharp 5 \overline{\mathbf{C P}}^{2}$.

2. One expects that for any relative prime integers $p$ and $q$, there is an embedding $C_{p, q}$ in $E(n) \sharp k \overline{\mathbf{C P}}^{2}$, for some $k \in \mathbf{Z}$, such that the rational blow-down is diffeomorphic to $E(n ; p)$.

3. The key ingredient in the proof of the theorem is to find such a configuration $C_{k q-1, q}$. We chose $u_{i}$ exactly the same as $u_{i}$ embedded in $\sharp(k+q-2) \overline{\mathbf{C P}}^{2}$ except

$$
u_{k+q-3}=f-2 e_{1}-e_{2} \cdots-e_{q-1} \quad\left(u_{k-1}=f-2 e_{1}-e_{2}, \quad \text { if } q=2\right)
$$

4. One can extend the 'logarithmic transform' procedure to any 4-manifold which contains a cusp neighbourhood. A cusp in a 4-manifold means a PL embedded 2-sphere of self-intersection 0 with a single non-locally flat point whose neighbourhood is the cone on the right-hand trefoil knot, and we define a cusp neighbourhood in a 4-manifold to be a manifold $N$ obtained by performing 0 -framed surgery on the trefoil knot in the boundary of the 4-ball. Note that since the trefoil knot is a fibred knot with a genus 1 fibre, $N$ is fibred by tori with one singular fibre which is a cusp. Hence one can perform ' $p$-log transform' on a regular torus fibre in $N$ exactly the same way as in $E(n)$, so that the theorem above is also true for any smooth 4-manifold containing a cusp neighbourhood.

\section{Seiberg-Witten Theory of Rational Blow-downs of 4-Manifolds}

In this section we compute the Seiberg-Witten invariants of rational blow-downs of 4-manifolds. We start by recalling the basics of Seiberg-Witten invariants introduced by Seiberg and Witten (see $[\mathbf{8}, \mathbf{1 0}])$. 
Let $X$ be an oriented, closed Riemannian 4 -manifold, and let $L$ be a characteristic line bundle on $X$, that is, $c_{1}(L)$ is an integral lift of $w_{2}(X)$. This determines a Spin ${ }^{c}$. structure on $X$. We denote the associated $U(2)$-bundles by $W^{ \pm}:=S^{ \pm} \otimes L^{1 / 2}$, where $S^{ \pm}$is a (locally defined) spinor bundle on $X$. (One may choose a Spin ${ }^{c}$-structure first, and associated $U(2)$-bundles $W^{ \pm}$on $X$. Then $L:=\operatorname{det}\left(W^{+}\right) \cong \operatorname{det}\left(W^{-}\right)$is the associated characteristic line bundle on $X$.) For simplicity we assume that $H^{2}(X ; Z)$ has no 2-torsion so that the set $\operatorname{Spin}^{c}(X)$ of $\operatorname{Spin}^{c}$-structures on $X$ is identified with the set of characteristic line bundles on $X$.

Note that the Clifford multiplication $c: T^{*} X \rightarrow \operatorname{Hom}\left(W^{+}, W^{-}\right)$leads to an isomorphism

$$
\rho: \Lambda^{+} \otimes \mathbf{C} \longrightarrow \operatorname{sl}\left(W^{+}\right)
$$

taking $\Lambda^{+}$to $s u\left(W^{+}\right)$, and the Levi-Civita connection on $T X$ together with a unitary connection $A$ on $L$ induces a connection $\nabla_{A}: \Gamma\left(W^{+}\right) \rightarrow \Gamma\left(T^{*} X \otimes W^{+}\right)$. This connection, followed by Clifford multiplication, induces a Spin ${ }^{c}$-Dirac operator $D_{A}: \Gamma\left(W^{+}\right) \rightarrow \Gamma\left(W^{-}\right)$. The Seiberg-Witten equations [10] are the following pair of equations for a unitary connection $A$ of $L$ and a section $\Psi$ of $\Gamma\left(W^{+}\right)$:

$$
\left\{\begin{array}{l}
D_{A} \Psi=0 \\
\rho\left(F_{A}^{+}\right)=i\left(\Psi \otimes \Psi^{*}\right)_{0}
\end{array}\right.
$$

where $F_{A}^{+}$is the self-dual part of the curvature of $A$ and $\left(\Psi \otimes \Psi^{*}\right)_{0}$ is the trace-free part of $\left(\Psi \otimes \Psi^{*}\right)$ which is interpreted as an endomorphism of $W^{+}$.

The gauge group $\mathcal{G}:=\operatorname{Aut}(L) \cong \operatorname{Map}\left(X, S^{1}\right)$ acts on the space $\mathcal{A}_{X}(L) \times \Gamma\left(W^{+}\right)$ by

$$
g \cdot(A, \Psi)=\left(g \cdot A \cdot g^{-1}, g \cdot \Psi\right) .
$$

In particular, if $b_{1}(X)=0$, then the gauge group $\mathcal{G}$ is homotopy equivalent to $S^{1}$ so that the quotient

$$
\mathcal{B}_{X}^{*}(L):=\mathcal{A}_{X}(L) \times\left(\Gamma\left(W^{+}\right)-0\right) / S^{1}
$$

is homotopy equivalent to $\mathbf{C P}^{\infty}$. Since the set of solutions is invariant under the action, it induces an orbit space, called the (Seiberg-Witten) moduli space, denoted by $M_{X}(L)$, whose formal dimension is

$$
\operatorname{dim} M_{X}(L)=\frac{1}{4}\left(c_{1}(L)^{2}-3 \sigma(X)-2 e(X)\right)
$$

where $\sigma(X)$ is the signature of $X$ and $e(X)$ is the Euler characteristic of $X$.

Definition: A solution $(A, \Psi)$ of the Seiberg-Witten equation (2) is called irreducible (reducible) if $\Psi \neq \equiv 0(\Psi \equiv 0)$. 
Note that if $b^{+}(X)>0$ and $M_{X}(L) \neq \emptyset$, then for a generic metric on $X$ the moduli space $M_{X}(L)$ contains no reducible solutions, so that it is a compact, smooth manifold of the given dimension. Furthermore the moduli space $M_{X}(L)$ is orientable and its orientation is determined by a choice of orientation on $\operatorname{det}\left(H^{0}(X ; \mathbf{R}) \oplus H^{1}(X ; \mathbf{R})\right.$ $\left.\oplus H_{+}^{2}(X ; \mathbf{R})\right)$.

Definition: The Seiberg-Witten invariant for $X$ with $b_{1}(X)=0$ is the function $S W_{X}: \operatorname{Spin}^{c}(X) \rightarrow \mathbf{Z}$ defined by

$$
S W_{X}(L)= \begin{cases}0 & \text { if } \operatorname{dim} M_{X}(L)<0 \text { or odd } \\ \sum_{(A, \Psi) \in M_{X}(L)} \operatorname{sign}(A, \Psi) & \text { if } \operatorname{dim} M_{X}(L)=0 \\ \left\langle\beta^{d_{L}},\left[M_{X}(L)\right]\right\rangle & \text { if } \operatorname{dim} M_{X}(L):=2 d_{L}>0 \text { and even }\end{cases}
$$

where $\operatorname{sign}(A, \Psi)$ is \pm 1 whose sign is determined by an orientation on $M_{X}(L)$, and $\beta$ is a generator of $H^{2}\left(\mathcal{B}_{X}^{*}(L) ; \mathbf{Z}\right) \cong H^{2}\left(\mathbf{C P}^{\infty} ; \mathbf{Z}\right)$. For convenience, we denote the Seiberg-Witten invariant for $X$ by $S W_{X}=\sum_{L} S W_{X}(L) \cdot e^{L}$.

Note that if $b^{+}(X)>1$, the Seiberg-Witten invariant $S W_{X}=\sum S W_{X}(L) \cdot e^{L}$ is a diffeomorphism invariant, that is, $S W_{X}$ does not depend on the choice of generic metric on $X$ and generic perturbation of the Seiberg-Witten equation. Furthermore, only finitely many Spin $^{c}$-structures on $X$ have a non-zero Seiberg-Witten invariant.

Definition: Let $X$ be an oriented, smooth 4-manifold with $b_{1}=0$ and $b^{+}>1$. We say a cohomology class $c_{1}(L) \in H^{2}(X ; \mathbf{Z})$ is a Seiberg-Witten basic class (for brevity, $S W$-basic class) for $X$ if $S W_{X}(L) \neq 0$.

DEFINITION: An oriented, smooth 4-manifold $X$ is called a Seiberg-Witten simple type (for brevity, $S W$-simple type) if $S W_{X}(L)=0$, for all $L$ satisfying $\operatorname{dim} M_{X}(L)>0$.

Next we describe a (Seiberg-Witten) gluing theory for computing Seiberg-Witten invariants of a smooth 4-manifold $X=X_{+} \cup_{Y} X_{-}$which is separated into two pieces $X_{+}, X_{-}$by an embedded 3 -manifold $Y$. Let $\left(X_{R}, g_{R}\right)$ be the Riemannian manifold obtained from $X$ by cutting along $Y$ and inserting a cylinder $[-R, R] \times Y$ on which $g_{R}$ is a product metric. As in Donaldson theory, if the moduli space $M_{X_{R}}(L)$ is non-empty for all sufficiently large $R$, then by stretching the neck along $Y$ in $X$ (that is, $R \rightarrow \infty$ ) each solution $(A, \Psi) \in M_{X}(L)$ is split into three relative solutions

$$
\left(\left(A_{+}, \Psi_{+}\right),\left(A_{0}, \Psi_{0}\right),\left(A_{-}, \Psi_{-}\right)\right) \in M_{X_{+}}\left(\left.L\right|_{X_{+}}\right) \times M_{R \times Y}\left(\left.L\right|_{R \times Y}\right) \times M_{X_{-}}\left(\left.L\right|_{X_{-}}\right)
$$

and conversely any such three relative solutions $\left(A_{+}, \Psi_{+}\right),\left(A_{0}, \Psi_{0}\right)$ and $\left(A_{-}, \Psi_{-}\right)$induce a global solution $\left(A_{+}, \Psi_{+}\right) \sharp_{g_{1}}\left(A_{0}, \Psi_{0}\right) \sharp_{g_{2}}\left(A_{-}, \Psi_{-}\right) \in M_{X}(L)$, where $g_{1}$ and $g_{2}$ are gluing parameters. (In general, there is an obstruction to construct a global solution 
from relative solutions [3].) In particular, if the embedded 3 -manifold $Y$ in $X$ has a positive scalar curvature metric (for example, $Y=S^{3}, L\left(p^{2}, 1-p q\right)$ ), then any such solution $\left(A_{0}, \Psi_{0}\right) \in M_{R \times Y}\left(\left.L\right|_{R \times Y}\right)$ is reducible. That is,

$$
\begin{array}{r}
M_{R \times Y}\left(\left.L\right|_{R \times Y}\right)=\left\{\left(A_{0}, 0\right): A_{0} \text { is an ASD U(1) - connection on } Y\right\} \\
\\
\cong H^{1}(Y ; \mathbf{R}) / H^{1}(Y ; \mathbf{Z})
\end{array}
$$

For example, if $Y=S^{3}$ or $L\left(p^{2}, 1-p q\right)$, then $M_{R \times Y}\left(\left.L\right|_{R \times Y}\right)$ is a single reducible solution. Furthermore, since $L$ is a $U(1)$-bundle, the gluing parameters are $S^{1}$. In summary, we have

Proposition 3.1. If a smooth 4-manifold $X$ is split into two pieces $X_{+}$ and $X_{-}$by an embedded 3-manifold $Y=S^{3}$ or $L\left(p^{2}, 1-p q\right)$, then each solution $(A, \Psi) \in M_{X}(L)$ can be obtained from two relative solutions $\left(\left(A_{+}, \Psi_{+}\right),\left(A_{-}, \Psi_{-}\right)\right) \in$ $M_{X_{+}}\left(\left.L\right|_{X_{+}}\right) \times M_{X_{-}}\left(\left.L\right|_{X_{-}}\right)$and

$$
\operatorname{dim} M_{X}(L)=\operatorname{dim} M_{X_{+}}\left(\left.L\right|_{X_{+}}\right)+\operatorname{dim} M_{X_{-}}\left(\left.L\right|_{X_{-}}\right)+1
$$

where $M_{X_{i}}\left(\left.L\right|_{X_{i}}\right)$ is the set of solutions (modulo the gauge group) which converge asymptotically to a reducible solution in $M_{Y}\left(\left.L\right|_{Y}\right)$.

Note that if $\operatorname{dim} M_{X_{-}}\left(\left.L\right|_{X_{-}}\right)<0$, then $M_{X_{-}}\left(\left.L\right|_{X_{-}}\right)$consists of reducible solutions. The technical part in the rest of this section is to show that $\operatorname{dim} M_{B_{p, q}}\left(\left.L\right|_{B_{p, q}}\right)=$ -1 and $\operatorname{dim} M_{C_{p, q}}\left(\left.L\right|_{C_{p, q}}\right) \leqslant-1$, so that both $M_{B_{p, q}}\left(\left.L\right|_{B_{p, q}}\right)$ and $M_{C_{p, q}}\left(\left.L\right|_{C_{p, q}}\right)$ consist of a single reducible solution. Before doing this, as a warm-up, we can get a well-known blow-up formula [4] for Seiberg-Witten invariants by using index computations.

Proposition 3.2. If $X$ is a SW-simple type 4-manifold, then the blow-up $\widetilde{X} \equiv X \sharp \overline{\mathbf{C P}^{2}}$ is also of $S W$-simple type, and the Seiberg-Witten invariants of $\widetilde{X} \equiv$ $X \sharp \overline{\mathbf{C P}}^{2}$ are

$$
S W_{\widetilde{X}}=S W_{X} \cdot\left(e^{E}+e^{-E}\right)
$$

where $E$ is the exceptional divisor of $\overline{\mathbf{C P}}^{2}$.

Proof: Note that a characteristic line bundle on $\tilde{X} \equiv X \sharp \overline{\mathbf{C P}}^{2}$ is of the form $L+(2 k+1) E$, where $L$ is a characteristic line bundle on $X$ and $k \in \mathbf{Z}$. (We identify the exceptional divisor $E$ with its corresponding line bundle on $\overline{\mathbf{C P}}^{2}$.) Suppose $\tilde{L}:=$ $L+(2 k+1) E$ is a characteristic line bundle on $\tilde{X}$ such that $S W_{\widetilde{X}}(\tilde{L}) \neq 0$. Then, when splitting apart $\tilde{X}$ along $S^{3}$, Proposition 3.1 implies that any solution in $M_{\widetilde{X}}(\widetilde{L})$ can be obtained from two relative solutions which are identified with two (absolute) solutions in $M_{X}(L) \times M_{\overline{\mathbf{C P}}^{2}}((2 k+1) E)$. (Since stretching the neck along $S^{3}$ corresponds to 
choosing a sequence of metric so that the neck is pinched down to a point, the last statement follows from a simple removable singularities argument.) But since

$$
\begin{aligned}
\operatorname{dim} M_{\overline{\mathbf{C P}}^{2}}((2 k+1) E) & =\left.\left.2 \cdot \operatorname{ind} D_{A}\right|_{\overline{\mathbf{C P}}^{2}+\text { ind }\left(d^{+}+d^{*}\right)}\right|_{\overline{\mathbf{C P}}^{2}} \\
& =2 \cdot\left(e^{((2 k+1) E) / 2} \cdot \hat{A}\left(\overline{\mathbf{C P}}^{2}\right)\right) \cdot\left[\overline{\mathbf{C P}}^{2}\right]+\left(h^{1}-h^{0}-h^{+}\right)\left(\overline{\mathbf{C P}}^{2}\right) \\
& =2 \cdot \int_{\overline{\mathbf{C P}}^{2}}\left(\frac{((2 k+1) E)^{2}}{8}-\frac{p_{1}}{24}\right)-1 \\
& =2 \cdot \frac{-\left(4 k^{2}+4 k\right)}{8}-1 \\
& \leqslant-1 .
\end{aligned}
$$

(In case $Y=S^{3}$, ind $D_{A}$ has no boundary terms.) Thus $M_{\overline{\mathrm{CP}}^{2}}((2 k+1) E)$ consists of a single reducible solution, and $M_{\widetilde{X}}(\widetilde{L})$ can be identified with $M_{X}(L)$. Furthermore, since

$$
\begin{aligned}
\operatorname{dim} M_{\widetilde{X}}(\tilde{L}) & =\frac{1}{4}\left\{\left(c_{1}(L)+(2 k+1) E\right)^{2}-(3 \sigma(\tilde{X})+2 e(\tilde{X}))\right\} \\
& =\frac{1}{4}\left\{c_{1}(L)^{2}-(3 \sigma(X)+2 e(X))\right\}-\left(k^{2}+k\right) \\
& =\operatorname{dim} M_{X}(L)-\left(k^{2}+k\right),
\end{aligned}
$$

the $S W$-simple type condition on $X$ and $S W_{\widetilde{X}}(\widetilde{L}) \neq 0$ imply that $\operatorname{dim} M_{\widetilde{X}}(\widetilde{L})=0$ and $k=0$ or -1 . Hence $\widetilde{X}$ is also of $S W$-simple type and $S W_{X}(L)=S W_{\widetilde{X}}(L+E)=$ $S W_{\widetilde{X}}(L-E)$.

In order to compute ind $D_{A}$ on $B_{p, q}$ and $C_{p, q}$, we need the following two elementary trigonometric computations.

LEMma 3.1. For relatively prime integers $p$ and $q$, and $z=e^{(2 \pi i) / p^{2}}$

$$
\sum_{k=1}^{p^{2}-1} \frac{z^{t p k}}{\left(z^{k}-1\right)\left(z^{(p q-1) k}-1\right)}=\sum_{k=1}^{p^{2}-1} \frac{1}{\left(z^{k}-1\right)\left(z^{(p q-1) k}-1\right)}, \quad \text { for all } t \in \mathbf{Z} .
$$

ProOF: There exist integers $r$ and $s$ satisfying $r p+s q=1$; so $z^{t p k}=z^{s t p q k}$. Thus it suffices to show

$$
\sum_{k=1}^{p^{2}-1} \frac{z^{t p q k}-1}{\left(z^{k}-1\right)\left(z^{(p q-1) k}-1\right)}=0, \quad \text { for all } t \in \mathbf{Z}
$$


Given $t \in \mathbf{Z}$ and setting $w=z^{p q-1}$,

$$
\begin{aligned}
& \sum_{k=1}^{p^{2}-1} \frac{z^{(t+1) p q k}-z^{t p q k}}{\left(z^{k}-1\right)\left(z^{(p q-1) k}-1\right)} \\
& =\sum_{k=1}^{p^{2}-1} \frac{z^{t p q k}\left\{\left(z^{k}-1\right)\left(w^{k}-1\right)\right\}+z^{t p q k}\left\{\left(w^{k}-1\right)+\left(z^{k}-1\right)\right\}}{\left(z^{k}-1\right)\left(w^{k}-1\right)} \\
& =\sum_{k=1}^{p^{2}-1}\left\{z^{t p q k}+\frac{2}{\left(z^{k}-1\right)}\right\}+\sum_{k=1}^{p^{2}-1}\left\{\frac{\left(z^{t p q k}-1\right)}{\left(z^{k}-1\right)}+\frac{\left(w^{-(p q+1) t p q k}-1\right)}{\left(w^{k}-1\right)}\right\} \\
& =\sum_{k=1}^{p^{2}-1}\left\{z^{t p q k}+\frac{2}{\left(z^{k}-1\right)}\right\}+\sum_{k=1}^{p^{2}-1}\left\{\frac{\left(z^{t p q k}-1\right)}{\left(z^{k}-1\right)}-\frac{\left(w^{t p q k}-1\right)}{w^{t p q k}\left(w^{k}-1\right)}\right\} \\
& =\sum_{k=1}^{p^{2}-1}\left\{z^{t p q k}+\frac{2}{\left(z^{k}-1\right)}\right\}+\sum_{l=0}^{t p q-1} \sum_{k=1}^{p^{2}-1}\left\{z^{l k}-\left(w^{-1}\right)^{(t p q-l) k}\right\} \\
& =\sum_{k=1}^{p^{2}-1}\left\{z^{t p q k}+\frac{2}{\left(z^{k}-1\right)}\right\}+\sum_{l=0}^{t p q-1} \sum_{k=1}^{p^{2}-1} z^{l k}-\sum_{l=1}^{t p q} \sum_{k=1}^{p^{2}-1}\left(w^{-1}\right)^{l k} \\
& =\sum_{k=1}^{p^{2}-1}\left\{z^{t p q k}+\frac{2}{\left(z^{k}-1\right)}\right\}+\sum_{l=0}^{t p q-1} \sum_{k=1}^{p^{2}-1} z^{l k}-\sum_{l=1}^{t p q} \sum_{k=1}^{p^{2}-1} z^{l k} \\
& =\sum_{k=1}^{p^{2}-1} \frac{2}{\left(z^{k}-1\right)}+\left(p^{2}-1\right) \\
& =0 \text {. }
\end{aligned}
$$

Hence the lemma follows by induction on $t$.

LEMMA 3.2. For relatively prime integers $p$ and $q$, and $z=e^{(2 \pi i) / p^{2}}$

$$
\begin{aligned}
& s\left(1-p q, p^{2}\right)=\sum_{k=1}^{p^{2}-1} \cot \left(\frac{\pi k}{p^{2}}\right) \cdot \cot \left(\frac{\pi k(1-p q)}{p^{2}}\right)=\frac{2}{3}\left(1-p^{2}\right), \\
& \text { equivalently, } \sum_{k=1}^{p^{2}-1} \frac{1}{\left(z^{k}-1\right)\left(z^{(p q-1) k}-1\right)}=\frac{1}{12}\left(p^{2}-1\right)
\end{aligned}
$$

Note that this lemma can also be proved by using a different method [7].

Proof: An easy computation shows that

$$
s\left(1-p q, p^{2}\right)=\left(1-p^{2}\right)+\sum_{k=1}^{p^{2}-1} \frac{4}{\left(z^{k}-1\right)\left(z^{(p q-1) k}-1\right)}
$$


Note that for $0 \leqslant t \leqslant p-1$ and $w=z^{p}$,

$$
\begin{aligned}
\sum_{k=1}^{p-1} \frac{w^{t k}-1}{\left(w^{k}-1\right)\left(w^{-k}-1\right)} & =\sum_{l=0}^{t-1} \sum_{k=1}^{p-1} \frac{w^{l k}}{\left(w^{-k}-1\right)} \\
& =\sum_{k=1}^{p-1} \frac{-t}{\left(w^{k}-1\right)}-\sum_{l=1}^{t} \sum_{k=1}^{p-1} \frac{\left(w^{l k}-1\right)}{\left(w^{k}-1\right)} \\
& =\frac{t(p-1)}{2}-\sum_{l=1}^{t}((p-1)-(l-1)) \\
& =\frac{t^{2}-t p}{2}
\end{aligned}
$$

(The third equality follows from the fact that $\sum_{k=1}^{p-1} w^{l k}=-1$, for $1 \leqslant l \leqslant p-1$.) Hence by using the equality $\sum_{t=0}^{p-1} w^{t k}=0$ for $1 \leqslant k \leqslant p-1$,

$$
\begin{aligned}
0 & =\sum_{t=1}^{p-1} \sum_{k=1}^{p-1} \frac{w^{t k}}{\left(w^{k}-1\right)\left(w^{-k}-1\right)}+\sum_{k=1}^{p-1} \frac{1}{\left(w^{k}-1\right)\left(w^{-k}-1\right)} \\
& =\sum_{t=1}^{p-1} \frac{\left(t^{2}-t p\right)}{2}+\sum_{k=1}^{p-1} \frac{p}{\left(w^{k}-1\right)\left(w^{-k}-1\right)}
\end{aligned}
$$

so that

$$
\frac{p}{12}\left(p^{2}-1\right)=\sum_{k=1}^{p-1} \frac{p}{\left(w^{k}-1\right)\left(w^{-k}-1\right)} .
$$

Finally by using the fact that $\sum_{l=0}^{p-1} z^{l p q k}=0$ if $k \neq t p$ and $\sum_{l=0}^{p-1} z^{l p q k}=p$ if $k=t p$, and by Lemma 3.1, we have

$$
\begin{aligned}
\sum_{k=1}^{p^{2}-1} \frac{p}{\left(z^{k}-1\right)\left(z^{(p q-1) k}-1\right)} & =\sum_{l=0}^{p-1} \sum_{k=1}^{p^{2}-1} \frac{z^{l p k}}{\left(z^{k}-1\right)\left(z^{(p q-1) k}-1\right)} \\
& =\sum_{t=1}^{p-1} \frac{p}{\left(z^{t p}-1\right)\left(z^{(p q-1) t p}-1\right)} \\
& =\sum_{t=1}^{p-1} \frac{p}{\left(w^{t}-1\right)\left(w^{-t}-1\right)} \\
& =\frac{p}{12}\left(p^{2}-1\right) .
\end{aligned}
$$


PRoposition 3.3. For any characteristic line bundle $L_{B}$ on $B_{p, q}$ with a cylindrical end

$$
B_{p, q}^{+}=B_{p, q} \cup L\left(p^{2}, 1-p q\right) \times[1, \infty)
$$

$\operatorname{dim} M_{B_{p, q}^{+}}\left(L_{B}\right)=-1$; so the moduli space $M_{B_{p, q}^{+}}\left(L_{B}\right)$ consists of a single reducible solution.

Proof: It suffices to show that ind $\left(\left.D_{A}\right|_{B_{p, q}^{+}}\right)=0$ because

$$
\begin{aligned}
\operatorname{dim} M_{B_{p, q}^{+}}\left(L_{B}\right) & =2 \cdot \operatorname{ind}\left(\left.D_{A}\right|_{B_{p, q}^{+}}\right)+\left.\operatorname{ind}\left(d^{+}+d^{*}\right)\right|_{B_{p, q}^{+}} \\
& =2 \cdot \operatorname{ind}\left(\left.D_{A}\right|_{B_{p, q}^{+}}\right)+\left(b^{1}-b^{0}-b^{+}\right)\left(B_{p, q}^{+}\right) \\
& =2 \cdot \operatorname{ind}\left(\left.D_{A}\right|_{B_{p, q}^{+}}\right)-1
\end{aligned}
$$

where $A$ is a $U(1)$-connection on $L_{B} \rightarrow B_{p, q}^{+}$. Now compute

$$
\text { ind } \begin{aligned}
\left(\left.D_{A}\right|_{B_{p, q}^{+}}\right) & =\left(e^{\left(c_{1}\left(L_{B}\right)\right) / 2} \cdot \widehat{A}\left(B_{p, q}^{+}\right)\right) \cdot\left[B_{p, q}^{+}\right] \\
& =\int_{B_{p, q}^{+}}\left(\frac{c_{1}\left(L_{B}\right)^{2}}{8}-\frac{p_{1}}{24}\right)-\left(\frac{h+\eta(0)}{2}\right)
\end{aligned}
$$

Since $L_{B}$ is a flat connection on $B_{p, q}^{+}$the first term $\left(c_{1}\left(L_{B}\right)^{2}\right) / 8=0$, and the second term can be computed by using $[1$, Proposition 2.12$]$

$$
0=\sigma\left(B_{p, q}^{+}\right)=\int_{B_{p, q}^{+}}\left(\frac{p_{1}}{3}\right)+\frac{1}{p^{2}} \sum_{k=1}^{p^{2}-1} \cot \left(\frac{\pi k}{p^{2}}\right) \cdot \cot \left(\frac{\pi k(1-p q)}{p^{2}}\right) .
$$

Hence, by Lemma 3.2 ,

$$
\int_{B_{p, q}^{+}}\left(\frac{p_{1}}{24}\right)=\frac{-1}{8 p^{2}} \cdot s\left(1-p q, p^{2}\right)=\frac{1}{12 p^{2}}\left(p^{2}-1\right) .
$$

The boundary term, $(h+\eta(0)) / 2$, can also be computed by using the Atiyah-Singer fixed point theorem $\left[9\right.$, Section 19] for a $\operatorname{Spin}^{c}$-Dirac operator $D_{A}$ on $D^{4} / \mathbf{Z}_{p^{2}} \cong$ cone on $L\left(p^{2}, 1-p q\right)$ :

$$
\begin{aligned}
\frac{h+\eta(0)}{2} & =\frac{-1}{p^{2}} \sum_{g \in \mathbf{Z}_{p^{2}}-\{0\}} \operatorname{Spin}\left(g, D^{4}\right) \\
& =\frac{-1}{p^{2}} \sum_{k=1}^{p^{2}-1} \frac{\left(e^{\pi k i / p^{2}}-e^{-\pi k i / p^{2}}\right)\left(e^{(1-p q) \pi k i / p^{2}}-e^{-(1-p q) \pi k i / p^{2}}\right) \cdot e^{m p \cdot \pi k i / p^{2}}}{\left(1-e^{\pi k i / p^{2}}\right)\left(1-e^{-\pi k i / p^{2}}\right)\left(1-e^{(1-p q) \pi k i / p^{2}}\right)\left(1-e^{-(1-p q) \pi k i / p^{2}}\right)} \\
& =\frac{-1}{p^{2}} \sum_{k=1}^{p^{2}-1} \frac{e^{m p \cdot \pi k i / p^{2}}}{\left(e^{\pi k i / p^{2}}-e^{-\pi k i / p^{2}}\right)\left(e^{(1-p q) \pi k i / p^{2}}-e^{-(1-p q) \pi k i / p^{2}}\right)}
\end{aligned}
$$


where $c_{1}\left(\left.L_{B}\right|_{L\left(p^{2}, 1-p q\right)}\right)=m p \in H^{2}\left(L\left(p^{2}, 1-p q\right) ; \mathbf{Z}\right) \cong \mathbf{Z}_{p^{2}}$ (Lemma 2.2). Since $L_{B}$ is a characteristic line bundle, we can always choose an integer $m$ so that $m+q$ is even. (If $p$ and $m+q$ are odd, choose $m+p+q \equiv m+q(\bmod p)$. If $p$ is even, then $m$ and $q$ are odd.) By setting $z:=e^{2 \pi i / p^{2}}$ and $t:=(m+q) / 2 \in \mathbf{Z}$, we have

$$
\begin{array}{rlr}
\frac{h+\eta(0)}{2} & =\frac{-1}{p^{2}} \sum_{k=1}^{p^{2}-1} \frac{e^{\pi(m+q) k i / p}}{\left(e^{2 \pi k i / p^{2}}-1\right)\left(e^{2 \pi(p q-1) k i / p^{2}}-1\right)} \\
& =\frac{-1}{p^{2}} \sum_{k=1}^{p^{2}-1} \frac{z^{t p k}}{\left(z^{k}-1\right)\left(z^{(p q-1) k}-1\right)} \\
& =\frac{-1}{p^{2}} \sum_{k=1}^{p^{2}-1} \frac{1}{\left(z^{k}-1\right)\left(z^{(p q-1) k}-1\right)} \quad \text { (by Lemma 3.1) } \\
& =\frac{1}{12 p^{2}}\left(1-p^{2}\right) \quad \text { (by Lemma 3.2) }
\end{array}
$$

Combining these computations we get ind $\left(\left.D_{A}\right|_{B_{p}^{+},}\right)=0$.

Remark. In the proof of Proposition 3.3 above, if both $p$ and $m$ are even (in particular if $m=0$ ), a similar computation shows that ind $D_{A}$ on $B_{p, q}$ is not an integer. This contradiction means that $B_{p, q}$ is not spin for $p$ even (see Lemma 2.3).

Corollary 3.1. For any characteristic line bundle $L_{C}$ on $C_{p, q}^{+}=C_{p, q} \cup$ $L\left(p^{2}, 1-p q\right) \times[1, \infty), \operatorname{dim} M_{C_{p, q}^{+}}\left(L_{C}\right)$ is odd and $\leqslant-1 ;$ so the moduli space $M_{C_{p, q}^{+}}\left(L_{C}\right)$ consists of a single reducible solution.

Proof: Since ind $\left(d^{+}+\left.d^{*}\right|_{C_{p, q}^{+}}\right)=\left(b^{1}-b^{0}-b^{+}\right)\left(C_{p, q}^{+}\right)=-1$, in the same way as the proof above, it suffices to show that ind $\left(\left.D_{A}\right|_{C_{p, q}^{+}}\right) \leqslant 0$. Since $X=C_{p, q}^{+} \cup_{L} \overline{B_{p, q}^{+}}$ is homeomorphic to $\sharp k \overline{\mathbf{C P}}^{2}$ with $k=b_{2}\left(C_{p, q}\right)$, for any characteristic line bundle $L$ on $X, c_{1}(L)^{2} \leqslant-k$ and

$$
\text { ind }\left(\left.D_{A}\right|_{C_{p, q}^{+}}\right)+\operatorname{ind}\left(\left.D_{A}\right|_{B_{p, q}^{+}}\right)=\operatorname{ind}\left(D_{A} \mid X\right)=\int_{X} \frac{\left(c_{1}(L)^{2}+k\right)}{8} \leqslant 0 \text {. }
$$

Hence ind $\left(\left.D_{A}\right|_{C_{p, q}^{+}}\right) \leqslant-\operatorname{ind}\left(\left.D_{A}\right|_{B_{p, q}^{+}}\right)=0$.

Lemma 3.3. Let $X$ be a smooth 4-manifold containing a configuration $C_{p, q}$, that is, $X=X_{0} \cup_{L\left(p^{2}, 1-p q\right)} C_{p, q}$, and let $X_{p, q}$ be its rational blow-down. Then a line bundle $L$ on $X_{p, q}$ is characteristic if and only if both $\left.L\right|_{X_{0}}$ on $X_{0}$ and $\left.L\right|_{B_{p, q}}$ on $B_{p, q}$ are characteristic. 
Proof: Since $H^{1}\left(B_{p, q} ; \mathbf{Z}_{2}\right) \rightarrow H^{1}\left(L\left(p^{2}, 1-p q\right) ; \mathbf{Z}_{2}\right)$ is surjective, $i^{*} \oplus j^{*}$ : $H^{2}\left(X_{p, q} ; \mathbf{Z}_{2}\right) \rightarrow H^{2}\left(X_{0} ; \mathbf{Z}_{2}\right) \oplus H^{2}\left(B_{p, q} ; \mathbf{Z}_{2}\right)$ is injective. Hence the proof follows from the following commutative diagram

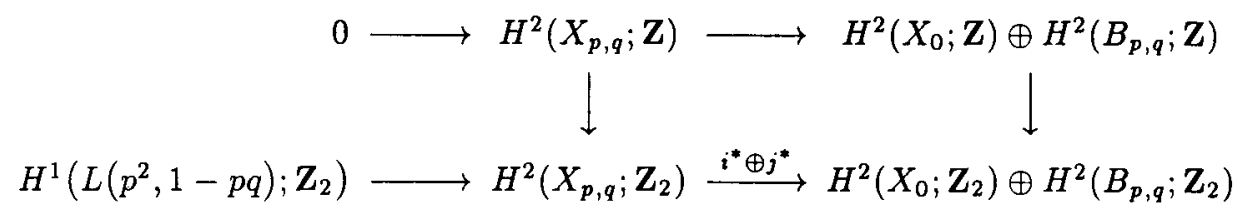

THEOREM 3.1. Suppose $X$ is a smooth 4-manifold which contains a configuration $C_{p, q}$. If $L$ is a characteristic line bundle on $X$ such that $S W_{X}(L) \neq 0$, $\left(\left.L\right|_{C_{p, q}}\right)^{2}=-b_{2}\left(C_{p, q}\right)$ and $c_{1}\left(\left.L\right|_{L\left(p^{2}, 1-p q\right)}\right)=m p \in \mathbf{Z}_{p^{2}} \cong H^{2}\left(L\left(p^{2}, 1-p q\right) ; \mathbf{Z}\right)$ with $m \equiv(p-1)(\bmod 2)$, then $L$ induces a characteristic line bundle $\bar{L}$ on $X_{p, q}$ such that $S W_{X_{p, q}}(\bar{L})=S W_{X}(L)$.

Proof: The condition $c_{1}\left(\left.L\right|_{L\left(p^{2}, 1-p q\right)}\right)=m p$ with $m \equiv(p-1)(\bmod 2)$ and Lemma 2.2 imply that the characteristic line bundle $\left.L\right|_{X_{0}}$ on $X_{0}$ extends uniquely to a characteristic line bundle $\bar{L}$ on $X_{p, q}$. Then the rest of proof is the same argument as the proof of [5, Theorem 8.2]. That is, first we study the solutions of Seiberg-Witten equations on $X$ for $L$ by pulling apart $X=X_{0} \cup_{L\left(p^{2}, 1-p q\right)} C_{p, q}$ along $L\left(p^{2}, 1-p q\right)$. Then Proposition 3.1 and Corollary 3.1 imply that each solution in $M_{X}(L)$ can be obtained by gluing a solution $\left(A_{X_{0}}, \Psi_{X_{0}}\right) \in M_{X_{0}}\left(\left.L\right|_{X_{0}}\right)$ with a unique reducible solution $\left(A_{C_{p, q}}, 0\right)=M_{C_{p, q}}\left(\left.L\right|_{C_{p, q}}\right)$. But, not every solution in $M_{X_{0}}\left(\left.L\right|_{X_{0}}\right)$ produces a global solution in $M_{X}(L)$. Explicitly, using Corollary 3.1, the inequality

$$
\begin{aligned}
2 d_{L}=\operatorname{dim} M_{X}(L) & =\operatorname{dim} M_{X_{0}}\left(\left.L\right|_{X_{0}}\right)+\operatorname{dim} M_{C_{p, q}}\left(\left.L\right|_{C_{p, q}}\right)+1 \\
& \leqslant \operatorname{dim} M_{X_{0}}\left(\left.L\right|_{X_{0}}\right)=2 d_{\left.L\right|_{X_{0}}}
\end{aligned}
$$

implies that there is an obstruction bundle $\xi$ of rank $d_{\left.L\right|_{X_{0}}}-d_{L}$ associated to the basepoint fibration over $M_{X_{0}}\left(\left.L\right|_{X_{0}}\right)$ such that the zero set of a generic section of $\xi$ is homologous to $M_{X}(L)$ in $\mathcal{B}_{X}^{*}(L)$ [3, Theorem 4.53], or [4, Section 4]. Hence

$S W_{X}(L)=\left\langle\beta^{d_{L}},\left[M_{X}(L)\right]\right\rangle=\left\langle\beta^{d_{L}}, \beta^{\left.d_{L}\right|_{X_{0}}-d_{L}} \cap\left[M_{X_{0}}\left(\left.L\right|_{X_{0}}\right)\right]\right\rangle=\left\langle\beta^{d_{L \mid} X_{0}},\left[M_{X_{0}}\left(\left.L\right|_{X_{0}}\right)\right]\right\rangle$

where $\beta$ is a generator of $H^{2}\left(\mathcal{B}_{X}^{*}(L) ; \mathbf{Z}\right)$. Similarly, since $\operatorname{dim} M_{B_{p, q}}\left(\left.\bar{L}\right|_{B_{p, q}}\right)=-1$ by Proposition 3.3 , the same argument as above shows

$$
S W_{X_{p, q}}(\bar{L})=\left\langle\beta^{\left.d_{L}\right|_{X_{0}}},\left[M_{X_{0}}\left(\left.L\right|_{X_{0}}\right)\right]\right\rangle
$$

so that $S W_{X_{p, q}}(\bar{L})=S W_{X}(L)$. 
COROLlary 3.2. If two characteristic line bundles $L$ and $L^{\prime}$ on $X$ satisfying the hypothesis in Theorem 3.1 induce the same characteristic line bundle $\bar{L}$ on $X_{p, q}$, then $S W_{X}(L)=S W_{X}\left(L^{\prime}\right)$.

Freedman's classification of simply connected topological 4-manifolds implies that $X \equiv C_{p, q} \cup_{L} \overline{B_{p, q}}$ is homeomorphic to $\sharp k \overline{\mathbf{C P}}^{2}$ with $k=b_{2}\left(C_{p, q}\right)$. Each generator $e_{i}$ of $H^{2}(X ; \mathbf{Z})$ when restricted to $B_{p, q}$ has the boundary value $\partial\left(\left.e_{i}\right|_{B_{p, q}}\right)=m p \epsilon$ $H^{2}\left(L\left(p^{2}, 1-p q\right) ; \mathbf{Z}\right)$ for some $m$. We impose the following condition $(*)$ on $C_{p, q}$ :

$\left(^{*}\right) \quad\left\{\partial\left(\left.\sum_{i=1}^{k} \varepsilon_{i} e_{i}\right|_{B_{p, q}}\right): \varepsilon_{i}= \pm 1, \forall i\right\}$

$$
=\{m p:-(p-1) \leqslant m \leqslant(p-1) \text { and } m \equiv(p-1)(\bmod 2)\} .
$$

All known configurations $C_{p, q}$ satisfy the condition (*) above. (One expects that all relatively prime integers $(p, q)$ satisfy the condition $(*)$.) Under this assumption, we prove

Lemma 3.4. Suppose $X$ is a simply connected smooth 4-manifold which contains a configuration $C_{p, q}$ satisfying the condition (*), and let $X_{p, q}$ be its rational blow-down. If $\bar{L}$ is a characteristic line bundle on $X_{p, q}$, there exists a characteristic line bundle $L$ on $X$ such that $\left.L\right|_{X_{0}}=\left.\bar{L}\right|_{X_{0}}$ and $c_{1}\left(\left.L\right|_{C_{p, q}}\right)^{2}=-k$, where $k=b_{2}\left(C_{p, q}\right)$.

Proof: The condition $(*)$ on $C_{p, q}$ implies that there exists $\varepsilon_{i}= \pm 1$, for $1 \leqslant$ $i \leqslant k$, such that $\partial\left(\left.\sum_{i=1}^{k} \varepsilon_{i} e_{i}\right|_{B_{p, q}}\right)=m p=\partial c_{1}\left(\left.\bar{L}\right|_{B_{p, q}}\right)$. Since the corresponding line bundle, denoted by the same notation $\sum_{i=1}^{k} \varepsilon_{i} e_{i}$, is characteristic on $C_{p, q} \cup_{L} \overline{B_{p, q}}$ which is homeomorphic to $\sharp k \overline{\mathbf{C P}}^{2}$, its restriction $\left.\sum_{i=1}^{k} \varepsilon_{i} e_{i}\right|_{C_{p, q}}$ is also characteristic on $C_{p, q}$ and $\left(\left.\sum_{i=1}^{k} \varepsilon_{i} e_{i}\right|_{C_{p, q}}\right)^{2}=\left(\sum_{i=1}^{k} \varepsilon_{i} e_{i}\right)^{2}-\left(\left.\sum_{i=1}^{k} \varepsilon_{i} e_{i}\right|_{\overline{B_{p, q}}}\right)^{2}=\left(\sum_{i=1}^{k} \varepsilon_{i} e_{i}\right)^{2}=-k$. Now define a line bundle $L$ on $X$ by

$$
L=\left\{\begin{array}{lll}
\left.\bar{L}\right|_{X_{0}} & \text { on } & X_{0} \\
\left.\sum_{i=1}^{k} \varepsilon_{i} e_{i}\right|_{C_{p, q}} & \text { on } & C_{p, q}
\end{array}\right.
$$

Then $L$ has the desired properties except (possibly) characteristic, that is, if $p$ is odd, then $L$ is automatically a characteristic line bundle on $X$, so we are done. If $p$ is even, we can change $L$ (see below) so that $L$ is characteristic on $X$ satisfying the same properties. 
Suppose $p$ is even.

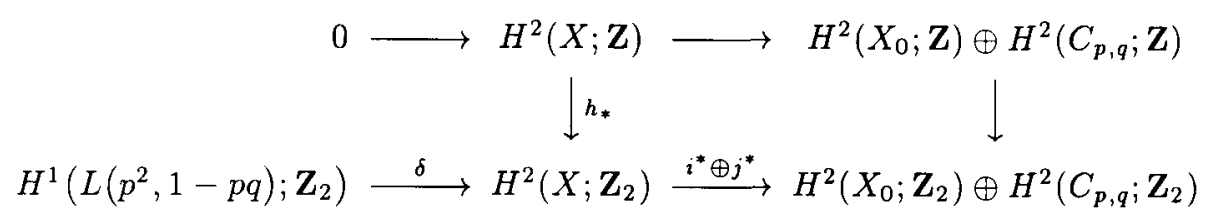

Since $X$ is simply connected, $H_{1}\left(X_{0} ; \mathbf{Z}\right) \cong \mathbf{Z}_{t}$ for some $t$ dividing $p^{2}$. If $t$ is even, then $i^{*} \oplus j^{*}: H^{2}\left(X ; \mathbf{Z}_{2}\right) \rightarrow H^{2}\left(X_{0} ; \mathbf{Z}_{2}\right) \oplus H^{2}\left(C_{p, q} ; \mathbf{Z}_{2}\right)$ is injective so that $L$ is characteristic. If $t$ is odd, then $i^{*} \oplus j^{*}$ is not injective, and in this case $h_{*}\left(c_{1}(L)\right)=w_{2}(X)$ or $w_{2}(X)+\delta(1)$.

Since $C_{p, q}$ satisfies the condition $(*)$, there exists $\delta_{i}= \pm 1$ satisfying $\left.\sum_{i=1}^{k} \delta_{i} e_{i}\right|_{p, q}=$ $(p-m) p$. Then setting $\gamma_{i} \equiv\left(\varepsilon_{i}+\delta_{i}\right) / 2$ we have
(1) $\partial\left(\left.\sum_{i=1}^{k} \gamma_{i} e_{i}\right|_{C_{p, q}}\right)=(p / 2) p \neq 0$,
(2) $\partial\left(\left.\sum_{i=1}^{k}\left(\varepsilon_{i}-2 \gamma_{i}\right) e_{i}\right|_{C_{p, q}}\right)=\partial\left(\left.\sum_{i=1}^{k} \varepsilon_{i} e_{i}\right|_{C_{p, q}}\right)=m p$,
(3) $\left.\sum_{i=1}^{k}\left(\varepsilon_{i}-2 \gamma_{i}\right) e_{i}\right|_{C_{p, q}}=\left.\sum_{i=1}^{k} \varepsilon_{i}^{\prime} e_{i}\right|_{C_{p, q}}$, for some $\varepsilon_{i}^{\prime}= \pm 1$.

Hence there exists a bundle $L^{\prime}$ on $X$ such that $\left.L^{\prime}\right|_{\lambda_{0}}=\left.L\right|_{X_{0}}$ and $\left.L^{\prime}\right|_{C_{p, q}}=$ $\left.\sum_{i=1}^{k}\left(\varepsilon_{i}-2 \gamma_{i}\right) e_{i}\right|_{C_{p, q}}$. Then we claim either $L$ or $L^{\prime}$ is ch racteristic: Suppose neither $L$ nor $L^{\prime}$ is characteristic, that is, $h_{*}\left(c_{1}(L)\right)=h_{*}\left(c_{1}\left(L^{\prime}\right)\right)=w_{2}(X)+\delta(1)$. Then $h_{*}\left(L-L^{\prime}\right)=0$, so that there exists an element $\alpha \in H^{2}(X ; \mathbf{Z})$ satisfying $2 \alpha=L-L^{\prime}$. Since both $H^{2}\left(X_{0} ; \mathbf{Z}\right)$ and $H^{2}\left(C_{p, q} ; \mathbf{Z}\right)$ are 2-torsion free,

$$
2\left(\left.\alpha\right|_{X_{0}},\left.\alpha\right|_{C_{p, q}}\right)=\left(i^{*} \oplus j^{*}\right)(2 \alpha)=\left(i^{*} \oplus j^{*}\right)\left(L-L^{\prime}\right)=2\left(0,\left.\sum_{i=1}^{k} \gamma_{i} e_{i}\right|_{C_{p, q}}\right)
$$

implies $\left.\alpha\right|_{X_{0}}=0$ and $\left.\alpha\right|_{C_{p, q}}=\left.\sum_{i=1}^{k} \gamma_{i} e_{i}\right|_{C_{p, q}}$ which contradicts $\partial\left(\left.\sum_{i=1}^{k} \gamma_{i} e_{i}\right|_{C_{p, q}}\right)=$ $(p / 2) p \neq 0$.

Finally, by using the same argument as in the proof of Theorem 3.1 with the characteristic line bundle $L$ on $X$ constructed in the Lemma 3.4 above, we get our main theorem.

THEOREM 3.2. If a simply connected smooth 4 -manifold $X$ contains a configuration $C_{p, q}$ satisfying the condition (*), then the Seiberg-Witten invariants of $X_{p, q}$ 
are completely determined by those of $X$. That is, for any characteristic line bundle $\bar{L}$ on $X_{p, q}$ with $S W_{X_{p, q}}(\bar{L}) \neq 0$, there exists a characteristic line bundle $L$ on $X$ such that $S W_{X}(L)=S W_{X_{p, q}}(\bar{L})$. Furthermore, if $X$ is of $S W$ - simple type, then $X_{p, q}$ is also of $S W$-simple type.

\section{EXAMPLES}

In this section we apply the result of the previous section to several examples of rational blow-downs. We compute the Seiberg-Witten invariants of a manifold constructed from $E(n)$ via blowing up and rationally blowing down.

Example 1. Consider a 4 -manifold $X \equiv E(3) \sharp 2 \overline{\mathbf{C P}}^{2}$ constructed by the following blowing up process (Figure 3 ):

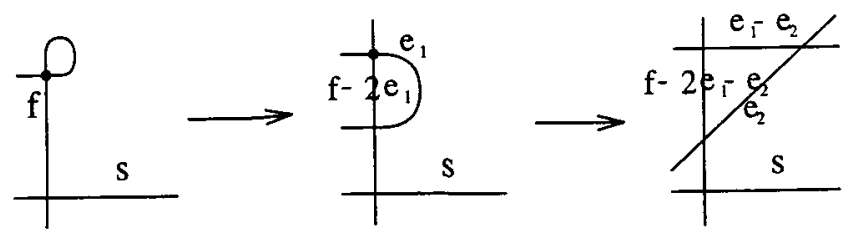

Figure 3

Then we get a configuration $C_{5,2} \subset X$

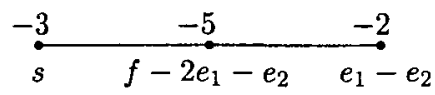

where $s$ is a section in $E(3)$ and $e_{i}(i=1,2)$ is the exceptional divisor in $\overline{\mathbf{C P}}^{2}$. Since $S W$-basic classes in $E(3)$ are $\pm f$, up to sign the $S W$-basic classes of $X$ are of the form

$$
L=f+\varepsilon_{1} e_{1}+\varepsilon_{2} e_{2} \quad\left(\varepsilon_{i}= \pm 1\right) .
$$

By using boundary values (see equation (1)), compute $\left.L\right|_{C_{5,2}}$ and $\partial\left(\left.L\right|_{C_{5,2}}\right)$

$$
\begin{aligned}
\left.L\right|_{C_{5,2}} & =\left(L \cdot u_{1}\right) \gamma_{1}+\left(L \cdot u_{2}\right) \gamma_{2}+\left(L \cdot u_{3}\right) \gamma_{3} \\
& =\left(\varepsilon_{2}-\varepsilon_{1}\right) \gamma_{1}+\left(2 \varepsilon_{1}+\varepsilon_{2}\right) \gamma_{2}+\gamma_{3}, \\
\partial\left(\left.L\right|_{C_{5,2}}\right) & =\left(\varepsilon_{2}-\varepsilon_{1}\right)+2\left(2 \varepsilon_{1}+\varepsilon_{2}\right)+9 \\
& =3\left(\varepsilon_{1}+\varepsilon_{2}\right)+9 .
\end{aligned}
$$

Then $\partial\left(\left.L\right|_{C_{5,2}}\right)$ is a multiple of $p=5$ if and only if $\varepsilon_{1}=\varepsilon_{2}=1$. Hence by Theorem 3.1, only $L=f+e_{1}+e_{2}$ descends to a $S W$-basic class $\bar{L}$ of $X_{5,2}$, and by Theorem $3.2, \bar{L}$ 
is the only $S W$-basic class of $X_{5,2}$. Since $c_{1}(\bar{L})^{2}=c_{1}(L)^{2}-c_{1}\left(\left.L\right|_{C_{5,2}}\right)^{2}=-2+3=1$, $X_{5,2}$ is a $S W$-simple type 4-manifold with $c_{1}^{2}=1$ which has one basic class $\bar{L}=$ $\overline{f+e_{1}+e_{2}}$ (up to sign) and its Seiberg-Witten invariant is $S W_{X_{5,2}}(\bar{L})=S W_{X}(L)=1$.

Next, let us consider a configuration $C_{4 q-1, q}$

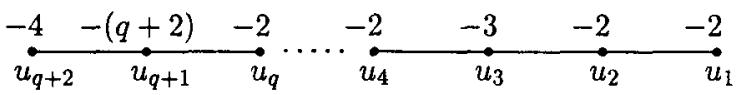

whose boundary values (see equation (1)) are given by

$$
\partial \gamma_{i}= \begin{cases}i & i=1,2 \\ 4 i-9 & i=3, \ldots, q+1 \\ (4 q-1) q-1 & i=q+2\end{cases}
$$

Then we have

Proposition 4.1. Suppose $X$ is a simply connected smooth 4 -manifold containing a configuration $C_{p, q}(p=4 q-1)$. If each $u_{i}$ satisfies $\left|L \cdot u_{i}\right|+u_{i}^{2} \leqslant-2$, for each basic class $L$ in $X$, then the Seiberg-Witten invariants of $X_{p, q}$ are given by $S W_{X_{p, q}}(\bar{L})= \begin{cases}S W_{X}(L) & \text { if } L \cdot u_{3}=\varepsilon, \quad L \cdot u_{q+1}=\varepsilon q \text { and } L \cdot u_{q+2}=2 \varepsilon \quad(\varepsilon= \pm 1) \\ 0 & \text { otherwise. }\end{cases}$ REMARK. The hypothesis, $\left|L \cdot u_{i}\right|+u_{i}^{2} \leqslant-2$, in Proposition 4.1 above comes from the adjunction inequality in [4]. Our assumption is that the $u_{i}$ are generic in the sense that they do not fall into the special case of $[4$, Theorem 1.3].

Proof: The condition $\left|L \cdot u_{i}\right|+u_{i}^{2} \leqslant-2$ implies $L \cdot u_{i}=0(i=1,2,4, \ldots, q)$, so that

$$
\begin{aligned}
\left.L\right|_{C_{p, q}} & =\left(L \cdot u_{3}\right) \gamma_{3}+\left(L \cdot u_{q+1}\right) \gamma_{q+1}+\left(L \cdot u_{q+2}\right) \gamma_{q+2} \\
\partial\left(\left.L\right|_{C_{p, q}}\right) & =3\left(L \cdot u_{3}\right)+(4 q-5)\left(L \cdot u_{q+1}\right)+(p q-1)\left(L \cdot u_{q+2}\right) \\
& \equiv 3\left(L \cdot u_{3}\right)-4\left(L \cdot u_{q+1}\right)-\left(L \cdot u_{q+2}\right)(\bmod p) .
\end{aligned}
$$

Since $\left.L\right|_{C_{p, q}}$ is characteristic, the condition $\partial\left(\left.L\right|_{C_{p, q}}\right) \equiv 0(\bmod p)$ in Theorem 3.1 implies that only basic class $\bar{L}$ in $X_{p, q}$ comes from $L$ of $X$ satisfying

$$
L \cdot u_{3}=\varepsilon, L \cdot u_{q+1}=\varepsilon q \text { and } L \cdot u_{q+2}=2 \varepsilon \quad(\varepsilon= \pm 1) .
$$

The rest of the proof follows from Theorem 3.2.

Example 2. Let $X \equiv E(q+2) \sharp 2 \overline{\mathbf{C P}}^{2}$ be a manifold constructed as follows: Consider the following configuration in $E(q+2)$

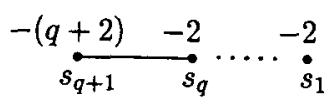


where $f \cdot s_{q+1}=1$ and $f \cdot s_{i}=0$, for $i=1, \cdots, q$. (One can choose such a configuration lying in the canonical resolution $Q$ of a singularity of $z_{1}^{2}+z_{2}^{2 q+3}+z_{3}^{4 q+5}=0$ in $\mathbf{C}^{3}$. Note that an elliptic surface $E(q+2)$, as a genus $q+1$ Lefschetz fibration, can be constructed as follows:

$$
E(q+2) \cong Q \cup_{\Sigma(2,2 q+3,4 q+5)} C(2,2 q+3) \sharp_{\Sigma} C(2,2 q+3) \cup_{\Sigma(2,2 q+3,4 q+5)} Q
$$

where $C(2,2 q+3)$ is a blow-up of the manifold obtained from +1 surgery on the $(2,2 q+3)$ torus knot and $\Sigma$ is an embedded surface of genus $q+1$ and self-intersection 0 in $C(2,2 q+3)$.) By blowing up the double point of a nodal fibre $f$ in $E(q+2)$ and a regular point in $s_{3}$, we have a configuration $C_{4 q-1, q} \subset X$ such that

$$
u_{q+2}=f-2 e_{1}, u_{3}=s_{3}-e_{2} \text { and } u_{i}=s_{i}, i \neq 3, q+2 .
$$

Since the $S W$-basic classes of $X$ have the form

$$
L=k f+\varepsilon_{1} e_{1}+\varepsilon_{2} e_{2}\left(|k| \leqslant q, k \equiv q(\bmod 2) \text { and } \varepsilon_{i}= \pm 1\right)
$$

this example satisfies the hypothesis of the Proposition 4.1 above. It follows that $X_{p, q}$ has one basic class $\bar{L}=\overline{q f+e_{1}+e_{2}}$ (up to sign) with $c_{1}\left(\bar{L}^{2}=q\right.$. Hence $X_{p, q}$ is a $S W$-simple type irreducible smooth 4-manifold lying in $c_{1}^{2}=\chi-2$ which has one basic class and cannot admit a complex structure.

EXAMple 3. ( $p$-log transform) As we see in [5] (or Theorem 2.1), $E(n ; p)$ is obtained by blowing up and rational blow-down from $E(n)$, so that the Seiberg-Witten invariants of $E(n ; p)$ can be computed explicitly as the same way as in Example 1:

THEOREM 4.1. ([5].) The Seiberg-Witten invariants of $E(n ; p)$ are

$$
S W_{E(n ; p)}=S W_{E(n)} \cdot\left(e^{(p-1) f_{p}}+e^{(p-3) f_{p}}+\cdots+e^{-(p-1) f_{p}}\right)
$$

where $f_{p}$ is a multiple fibre obtained by $p$-log transform on $E(n)$.

Furthermore, by extending the notion of ' $p$-log transform' to any smooth 4manifold containing a cusp neighbourhood, we extend this result.

COROLlary 4.1. Let $X(p)$ be the result of $p$-log transform in the neighbourhood of a cusp, say $f$, in a $S W$-simple type irreducible 4-manifold $X$. Then the Seiberg-Witten invariants of $X(p)$ are

$$
S W_{X(p)}=S W_{X} \cdot\left(e^{(p-1) f_{p}}+e^{(p-3) f_{p}}+\cdots+e^{-(p-1) f_{p}}\right)
$$

where $f_{p}$ is a multiple fibre in $X(p)$ obtained by $p$-log transform on $X$.

Proof: It suffices to show that $f \cdot L=0$ for each basic class $L$ of $X$. Since genus $(f)=1$ and $f^{2}=0$, this is implied by the adjunction inequality

$$
f^{2}+|f \cdot L| \leqslant 2 \cdot \operatorname{genus}(f)-2 .
$$

We close this paper by suggesting that Corollary 4.1 allows us to answer partially the uniqueness problems of irreducible 4-manifolds. 


\title{
REFERENCES
}

[1] M. Atiyah, V. Patodi, and I. Singer, 'Spectral asymmetry and Riemannian geometry II', Math. Proc. Cambridge. Philos. Soc. 78 (1975), 405-432.

[2] A. Casson and J. Harer, 'Some homology lens spaces which bound rational homology balls', Pacific J. Math. 96 (1981), 23-36.

[3] S. Donaldson, 'Connections, cohomology and the intersection forms of 4-manifolds', $J$. Differential Geom. 24 (1986), 275-341.

[4] R. Fintushel and R. Stern, 'Immersed spheres in 4-manifolds and the immersed Thom conjecture', Turkish J. Math. 19 (1995), 145-157.

[5] R. Fintushel and R. Stern, 'Rational blowdowns of smooth 4-manifolds', (MSRI-reprints: alg-geom/9505018), J. Differential Geom. (to appear).

[6] R. Gompf, 'Nuclei of elliptic surfaces', Topology 30 (1991), 479-511.

[7] F. Hirzebruch and D. Zagier, The Atiyah-Singer Theorem and elementary number theory, Mathematical Lecture Series 3 (Publish or Perish, Berkeley, 1974).

[8] P. Kronheimer and T. Mrowka, 'The genus of embedded surfaces in the projective plane', Math. Res. Lett. 1 (1994), 797-808.

[9] P. Shanahan, The Atiyah-Singer Index Theorem, Lecture Notes in Mathematics 638 (Springer-Verlag, Berlin, Heidelberg, New York, 1976).

[10] E. Witten, 'Monopoles and four-manifolds', Math. Res. Lett. 1 (1994), 769-796.

\author{
Department of Mathematics \\ Kon-kuk University \\ Kwangjin-gu Mojin-dong 93-1 \\ Seoul 143-701 \\ Korea \\ e-mail: jipark@kkucc.konkuk.ac.kr
}

